\title{
DISCAPACIDAD: UN ASUNTO DE JUSTICIA SOCIAL
}

\section{Disability: a Matter of Social Justice}

\author{
Delfín Ignacio Grueso VANegas \\ Universidad del Valle. Cali. Colombia \\ delfin.grueso@correounivalle.edu.co \\ Linda Marcela SAndoval Moreno \\ Universidad del Valle. Cali. Colombia
}

Recepción: 13 de enero de 2020

Aceptación: 4 de marzo de 2021

Resumen: Este artículo presenta la discapacidad como un asunto de Justicia Social, un problema moral a cuyo entendimiento aporta luces el Enfoque de las Capacidades (especialmente en la versión de Amartya Sen), que permite ver la 'discapacidad' en términos de mayor o menor libertad. Un paso crucial para entender esa mayor o menor libertad, es comenzar reconociendo el carácter histórico del espacio socialmente compartido en que viven las personas en discapacidad; en principio el espacio vital disponible para el desarrollo y expresión de la vida humana. En cada momento de la historia, ése es un espacio cultural y tecnológicamente constituido y arroja como resultado diferencial las condiciones de 'capacidad' y 'discapacidad' en las que se encuentran los seres humanos para el disfrute de su libertad. Es del carácter de 'hecho social' que finalmente tiene ese espacio que emerge la discapacidad como un efecto diferencial del mismo y se constituye en un problema moral que debe ser afrontado desde una perspectiva normativa, la de la justicia social.

Palabras clave: discapacidad; capacidad; Justicia Social; Enfoque de las Capacidades.

AвSTRACT: This article presents disability as a Social Justice issue, a moral problem whose understanding is illuminated by the Capabilities Approach (especially in Amartya Sen's version), which allows us to see 'disability' in terms of greater or lesser freedom. A crucial step to understand this greater or lesser freedom is to begin by recognizing the historical character of the socially shared space in which people with disabilities live; in principle the vital space available for the development and expression of human 
life. At each moment in history, this is a culturally and technologically constituted space and it gives as a differential result the conditions of 'capacity' and 'disability' in which human beings find themselves to enjoy their freedom. It is of the character of 'social fact' that this space finally has that disability emerges as a differential effect of it and becomes a moral problem that must be faced from a normative perspective, that of social justice.

KEYwORDs: disability; capacity; Social Justice; Capabilities Approach.

\section{Introducción}

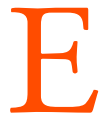

L FENÓMENO DE LA DISCAPACIDAD -ampliamente estudiado durante los siglos XIX y XX-gana mucho en perspectiva ético-política si se lo trata en términos de la Justicia Social. Es lo que este artículo hace, apelando principalmente al enfoque de las capacidades (sobre todo en la versión de Amartya Sen); liga la justicia a la discapacidad a través de la libertad; de la mayor o menor libertad de un individuo para alcanzar las metas que se propone y para vivir funcionalmente en una sociedad determinada.

Un aspecto central del artículo es mostrar cómo la discapacidad se constituye en una limitación de la libertad de elección de un individuo no solo por una condición específica corporal de él, sino, ante todo, por la relación que esa condición guarda con disposiciones contextuales para el desarrollo de la vida de los seres humanos; disposiciones que terminan constituyendo en barreras. En otras palabras, una condición de discapacidad emerge a partir de cierta construcción social del espacio vital, es decir, aquel abierto para el desarrollo y expresión de la vida humana; un espacio forjado a partir de presupuestos y condiciones propios de un contexto social, tecnológica e históricamente determinado. En un espacio así, la condición de discapacidad emerge como una situación de menor libertad. Es en relación con esta situación de 'menor libertad' que cobra sentido la conexión de la discapacidad con el Enfoque de Capacidades. Se tratará de mostrar esta menor libertad como un problema de justicia social.

El artículo concluye que ese enfoque está lejos de iluminar una solución justiciera más comprehensiva. La razón es que dicho enfoque no ahonda suficientemente en el tratamiento de los otros determinantes sociales (determinantes que a su vez requieren ser abordados desde una perspectiva más estructural de la injusticia social; perspectiva que algunos echan de menos en Sen), las condiciones en las que las personas nacen, crecen, viven y desarrollan sus vidas y el modo en que las mismas alivian o agravan la condición de discapacidad; en lo que él no se destaca es el tratamiento más estructural, en términos de justicia social, de las condiciones de origen de las injusticias sociales; uno que le permita iluminar, de una manera más comprehensiva, una transformación más profunda de la sociedad. Esto cobra especial relevancia en el tratamiento de las injusticias ligadas al fenómeno de la discapacidad, cuya superación requiere una más sostenida transformación social y política (no apenas puntuales normas incorporadas en políticas públicas sectoriales) para remover de una manera eficaz y permanente las barreras espaciales, educativas, laborales, entre otras, que arrojan a un lado 
a ciertos individuos en tanto van incluyendo a otros en el espacio compartido y en la competencia por la vida. Sin esa transformación más profunda, más orientada a la transformación de la estructura social, no es posible una satisfactoria superación de la condición de discapacidad; sin una remoción de las otras facetas de la inequidad social (génesis estructurante de los 'determinantes sociales'), no se neutralizarán las condiciones que precipitan al individuo en 'condición de discapacidad' en una situación de "postración social”.

Una vez el artículo tematice la discapacidad como un asunto de menor libertad ligado a la construcción social del espacio vital, podrá avanzar presentándola como un problema de justicia social con una connotación política en el sentido más amplio de la palabra. Siendo esa la meta, y teniendo en ella cierta centralidad la libertad, tal y como está pensada en el enfoque ya mencionado, se hace indispensable el modo en que la libertad ya había ganado centralidad en la teoría de la justicia de John Rawls. Se espera que el contraste entre Rawls y Sen a propósito de la libertad ayude al tratamiento de la discapacidad como una cuestión de menor libertad. En el curso de ese análisis, se irá articulando el caso de Pedro y Juan, un recurso heurístico para mostrar el nexo entre tecnologías (educativas, rehabilitadoras) y discapacidad, por un lado, y determinantes sociales y discapacidad, por el otro.

\section{Discapacidad, espacio y libertad}

A fin de ir aclarando el nexo entre discapacidad y libertad, conviene avanzar algunas ideas generales sobre el espacio, su naturaleza histórica y social y su relación con los cuerpos y con la libertad humana; ideas que no provienen de la teoría seniana. La primera de esas ideas tiene que ver con el entendimiento que llega a tenerse, en cualquier contexto histórico y cultural, de lo que es y debe ser la vida humana; entendimiento al cual va ligada una lista de las competencias (si así puede llamárselas) que una persona debe alcanzar para desarrollar una forma de vida socialmente aceptable. Esto es importante porque esa base de criterios socialmente compartidos se traduce en el estímulo para el desarrollo de ciertas habilidades, destrezas y mecanismos adaptativos, considerados vitales para el desempeño funcional. En concreto, en diferentes culturas y en diferentes momentos históricos, los seres humanos son estimulados a desarrollar su vida de una forma determinada y en tanto alcancen ese desarrollo su desempeño funcional es socialmente valorado como exitoso.

Lo anterior permite avanzar otra idea sobre el nexo entre un determinado entendimiento sobre el desempeño funcional de la vida humana y el apropiamiento del espacio; porque también el espacio (el espacio de habitación, de movilidad, de trabajo) se configura histórica y culturalmente. El espacio, en general, se configura y reconfigura tecnológicamente para satisfacer unas expectativas socialmente compartidas en relación con la movilidad, la producción, el consumo y el desarrollo de las capacidades y el goce de la vida humana. Ese espacio, cualquier espacio histórica y tecnológicamente adecuado para la vivencia humana, determina el nivel de desarrollo funcional de todas las personas (con relativa independencia de su propio cuerpo y de sus capacidades 
físicas y mentales). Lo hace en la medida justa en que determina la capacidad de movilidad y de inserción de los individuos en las dinámicas de interacción social. La conclusión parcial de esto es que un individuo deviene en 'condición de discapacidad' en buena parte en virtud de disposiciones espaciales y de las herramientas comunes adaptadas para las necesidades de encuentro de las 'vidas humanas socialmente funcionales'. Esto es lo que significa decir que una condición de discapacidad emerge como resultado de la construcción social; que es un fenómeno histórico y social, no únicamente el resultado de la relación que un individuo tiene con su propio cuerpo.

Así las cosas, una condición de discapacidad no se reduce a la alteración estructural de algún órgano o a una variación específica en la funcionalidad del organismo de un individuo ni a la relación que él/ella pueda llegar a alcanzar con su propio cuerpo, sino que se configura en el encuentro entre una condición corporal y las condiciones del ambiente en el cual el sujeto se ve obligado a desenvolverse. Hasta cierto punto, es un efecto del juego de relaciones de las habilidades corporales y cognitivas, de un lado, y, del otro, de juicios de los otros individuos con un entorno dado; un entorno forjado a partir de un sistema político, cultural, económico, social y educativo específico.

Ahora bien: puesto que cada sistema tiende a educar a la gente de cierta manera y a disponer de cierta manera el espacio y las herramientas para el trabajo y la movilidad de los individuos, el sujeto con discapacidad emerge como un efecto diferenciado entre individuos que 'deberían ser iguales', pero que no lo son: algunos acusan ciertas diferencias corporales, con lo que resultan teniendo una 'desventaja comparativa' (adicional en muchos casos a las desventajas comparativas emergentes de los determinantes sociales de clase social, género, 'raza', etc.) con respecto al resto de la sociedad. A esa condición en la que terminan encontrándose ciertos individuos como consecuencia de estos juegos relacionales, podemos identificarla como un 'déficit de libertad'. Déficit social e históricamente creado y expresado en una disminución de las posibilidades de autodeterminación; déficit en tanto establece y potencia la discapacidad de los individuos.

Ya debería quedar claro por qué, en virtud de esa condición espacial socialmente compartida (histórica, cultural, tecnológicamente creada), la discapacidad trasciende la relación del individuo con su propio cuerpo y deviene 'carga' (para el individuo y para sus semejantes), generando una no-libertad. Es una carga porque, en virtud de las lógicas imperantes en cada contexto para el logro de la movilidad, el desempeño y la autodeterminación individual, el individuo no es tan libre como otros para forjarse metas y ponerse en acción al mismo nivel que los otros, para alcanzarlas. Y dado que una discapacidad termina siendo envolvente en virtud de una determinación económico-política o simbólico-cultural de la vida en común, la cuestión no se resuelve por medios exclusivamente terapéuticos; va más allá de los avances en las terapias rehabilitadoras o de la disposición de nuevas tecnologías o distribuciones espaciales y herramientas más benignas.

¿Cómo conectar esta menor libertad con la noción de injusticia social? Ante todo, resaltando la naturaleza social del fenómeno de la discapacidad; mostrando que no se reduce a una 'condición natural'; resaltando el hecho de que la discapacidad está determinada por el encuentro entre una condición corporal especial (congénita o ad- 
quirida) y una serie de disposiciones y barreras socialmente creadas. Siendo este encuentro socialmente condicionado, no puede ser tratado como un hecho que le ocurre al individuo de forma aislada, que es 'su problema', algo que él debe resolver por sí mismo o resignarse a vivirlo como 'desgracia personal'.

Ese es el nexo entre la 'condición de discapacidad', tomada como un hecho social y valorada como una 'injusticia social', y la justicia como un valor moral que la sociedad debe tratar de alcanzar a través de nuevos arreglos sociales; acuerdos de naturaleza ético-política, algo en lo que coinciden Rawls y Sen ${ }^{1}$. Aplicados a la tarea de la superación de la condición de discapacidad, estos arreglos suponen acuerdos que deben extenderse hasta alcanzar la remoción o mitigación de disposiciones espaciales y complejidades técnicamente desarrolladas que, pensadas para el flujo, desarrollo y expresión de las diversidades de facultades humanas, afectan diferenciadamente al individuo en condición de discapacidad. De ser alcanzados, esos acuerdos ético-políticos deben ser traducidos en principios reguladores capaces de corregir las asimetrías que afectan, de una manera diferenciada, a las personas con discapacidad (condición que emerge como 'atributo diferencial', si así puede decirse).

La superación de esta injusticia tiene, en última instancia, una dimensión política. La tiene, para comenzar, porque, siendo la condición de discapacidad un hecho socialmente construido, es también socialmente cambiable. ¿Qué quiere decir esto? Ante todo, que no tiene que ser necesariamente así, especialmente desde que los desarrollos educativos, políticos, médicos y tecnológicos permiten organizar la sociedad y el espacio social de otro modo. Pero, ante todo, porque en la medida en que la sociedad adquiera el compromiso moral para alcanzar condiciones de equidad para la vida y el despliegue de las facultades humanas, queda compelida a remover o mitigar las disposiciones que, cada vez más sin necesidad, entorpecen el desarrollo vital de ciertos individuos. No puede satisfacer ese compromiso moral sin alcanzar acuerdos para la reorganización institucional de la sociedad que, aparte de ser moralmente justificados, sean viables; que no queden atrapados en el plano del deber ser, sino que, incorporados en políticas públicas, sean capaces de generar efectos a mediano plazo en el ordenamiento social.

Pero esto es además un asunto político de primer orden, además, porque existen otros condicionamientos sociales que incrementan la "carga" en discapacidad y mantienen el ya referido déficit de libertad. Esa carga y ese déficit son sin duda más difíciles de superar para los pobres, los desempleados y las mujeres, para los sectores 'racializados', los individuos sexualmente diferenciados o estigmatizados en razón de su orientación sexual, para las minorías religiosas y culturales, en fin, para esos grupos o sectores poblacionales 'subordinados' por cierta estructuración socioeconómica y simbólico-cultural dominante de la sociedad. A las desventajas estructurales que su-

\footnotetext{
1 Para el caso de Sen, valga la pena mencionar esto que dice en relación con la equidad en salud: "La equidad en salud no concierne únicamente a la salud, vista aisladamente, sino que debe abordarse desde el ámbito más amplio de la imparcialidad y la justicia de los acuerdos sociales, incluida la distribución económica, y prestando la debida atención al papel de la salud en la vida y la libertad humanas. Ciertamente, la equidad en salud no trata solo de la distribución de la salud, por no hablar del campo todavía más limitado de la distribución de la asistencia sanitaria" (Sen, 2002, p. 302).
} 
fre, en razón de su 'pertenencia' a un grupo subordinado, se le suman las relacionadas con la alteración funcional de algún órgano de su cuerpo y ambas se conjugan agrandando aún más su subordinación. En este cruce de determinaciones, este específico individuo acusa un plus de afectación; está afectado por esas determinaciones de un modo particular (y particularmente agravado).

Como ya se insinuó, el cruce de esta doble subordinación remite a cuestiones ético-políticas de primer orden: cuestiones radicalmente políticas porque, en última instancia, dependen del cambio de vocación social en relación con lo que significa igual libertad para todos a lo largo del espectro social. Sin esa nueva vocación no se alcanzarán las transformaciones que se necesitan en el espacio común, en el sistema educativo, laboral y de recreación, para superar la injusticia de esta doble subordinación.

De esta manera debería quedar claro por qué la cuestión de la justicia social para las personas en condición de discapacidad debe ser llevada al plano político: porque depende, en última instancia, de arreglos políticos básicos para la organización de la vida en común. Si la remoción de las barreras específicas que afectan a la población con una acusada condición de discapacidad depende de la remoción de las otras formas estructurales de subordinación social, de procesos que son principalmente políticos, esta cuestión de justicia social alcanza claramente la dimensión política que, en el límite, supone la erradicación de cualquier injusticia estructural.

Como una conclusión parcial de este acápite, habría que decir que, más allá de la solidaridad interpersonal, del desarrollo de más sofisticadas terapias, de puntuales reacomodos espaciales y en los procesos educativos, buscando puntuales formas de inserción de las personas en condición de discapacidad en las relaciones sociales donde todos desarrollan su proyecto y estilo de vida, al final todo se remite al nivel de acogida de esta problemática en los procesos de formación de la opinión y la voluntad política, esto es, la transformación política de la conciencia social y de la acción pública que reconduce la administración política de la vida en común.

Dicho esto, avancemos un paso más en nuestra argumentación, conectando discapacidad, libertad y justicia.

\section{Discapacidad y justicia social: el enfoque de las capacidades frente a Rawls}

La consideración de cómo el espacio vital es, en última instancia, una construcción sociohistórica ya debería ponernos a salvo de tratar la discapacidad en términos de 'desgracia'; de algo que le ocurre al individuo sin que este ni nadie a su alrededor tenga responsabilidad alguna y que, en razón de eso, la consideración social de su situación no puede ser otra cosa que un acto de caridad o compasión. Suficientemente nos ha mostrado Judith Shklar cómo las fronteras entre desgracia e injusticia se van moviendo según se va alterando en la historia la intervención humana en 'lo natural' al tenor del desarrollo de la ciencia y la tecnología, del cambio en las concepciones de mundo y del entendimiento de cómo debería ser reorganizada políticamente la vida social. En la medida en que hemos venido asistiendo a esos cambios, la discapacidad no puede 
quedar al margen de la justicia social, como 'lo simplemente dado', una fatal realidad a la que toca resignarse. La pasividad frente a la discapacidad puede ser el resultado de una cómoda interpretación ${ }^{2}$ (Shklar, 1990).

El punto es que, ante lo que podríamos todavía llamar una desgracia, ante algo que nosotros no hemos generado, ni como individuos ni como sociedad, no emerge una obligación de justicia; si acaso un sentimiento de compasión o una actitud de indiferencia que no puede ser, en sentido estricto, moralmente censurada. La injusticia, en cambio, nos cuestiona como sociedad. En esto es clave el reconocimiento que hace John Rawls de la naturaleza fortuita de ciertas desventajas con las que nacemos ('lotería natural' llama a ese hecho de la gratuidad congénita o genética) y la afirmación, aunque parte de reconocer que esas desventajas no pueden quedar al margen de la justicia social, al mismo tiempo tiene la idea de que frente a ellas no podemos cruzarnos de brazos. En sus palabras, es claro que "la distribución natural no es ni justa ni injusta... Lo que puede ser justo o injusto es el modo en que las instituciones actúan respecto a estos hechos" (Rawls, 1997).

Esta es una poderosa intuición. No obstante, pese a las variadas conexiones que se hacen de la teoría de la justicia de John Rawls, llamada por este justicia como equidad, y el campo de la salud ${ }^{3}$, es evidente que no hay en el primer Rawls, el de Una teoría de la justicia, publicado en 1971, una preocupación especial por este campo ${ }^{4}$. Bien es verdad que a través de sus dos principios de justicia y del 'principio de diferencia', aspecto clave del segundo principio, se pueden encontrar conexiones entre la salud y la igualdad de oportunidades que debe exhibir una sociedad para poder decir, de sí misma, que es una sociedad justa. También es verdad que la conexión también puede hallarse por el lado de la noción de 'bienes primarios' (que es justamente aquella privilegiada por Amartya Sen), para alcanzar una conexión con la libertad y la igualdad de oportunidades. Pero, de nuevo, son más evidentes las implicaciones de esa teoría en términos económico-políticos, al modo clásico de las teorías redistributivas de la

2 Dice Judith Shklar: "Nuestra disposición a actuar (ante una injusticia) está implicada aquí, en la medida en que a menudo tenemos que decidir si se ha producido o una injusticia, y siempre existe una fuerte tendencia a no hacer nada si es posible. Todos somos proclives a ser pasivamente injustos. La propia distinción entre injusticia y desventura a veces puede ser esquiva, inducir a hacer demasiado o a hacer poco" (Shklar, 2010).

3 Ejemplos de esas conexiones se hallan en obras tan tempranas como los capítulos de Walzer en Las esferas de la justicia; o en "El estudio empírico de la justicia”, de Jon Elster; o en Thoward a Healthy Society. The Morality and Politics of American Health Care Reform, de Milton Fisk; o el capítulo de Norman Daniels, Bruce Kennedy y Achiro Kawachi, "Health and Inequality, or, Why Justice is Good for Our Health", o "Justice in Health Care: Reflections on the Foundations of Health Equality", de Thomas Pogge, entre otros (ver Referencias al final). Todos ellos, de un modo u otro, tratan la conexión entre justicia y salud en la senda abierta por Rawls, pero yendo a donde su propia teoría no fue.

4 Para un entendimiento general de la teoría de la justicia del primer Rawls, ver Grueso (1997). Si Liberalismo politico no es privilegiado por quienes tratan los temas de la justicia en 'clave rawlsiana' (por ejemplo, Thomas Pogge), menos aún lo son otras obras que se alejan de algún modo del marco problemático propio de Una teoría de la justicia. Una obra como Justice as Fairness. A Restatement, por ejemplo, que ni siquiera es cabalmente una obra de Rawls (en verdad es más una adecuación alcanzada por Erin Kelly a partir de las propias reformulaciones de Rawls después de su obra cumbre de 1971), no aporta mucho en esta dirección. Una versión castellana de esta obra, donde puede verificarse esto, es La justicia como equidad. Una reformulación. Paidós, 2002. 
justicia, marcadas por la impronta aristotélica de alcanzar la equidad a través de un equilibrio entre capacidades, necesidades y méritos. Casi todos los autores que extrapolan los principios de justicia para tratar asuntos de salud y de políticas públicas en salud, lo hacen a partir del primer Rawls, el de Una teoría de la justicia. Tienen razón: el segundo Rawls, el de Liberalismo político, publicado en 1993, no vuelve a ahondar en los temas de la justicia social en el sentido tradicional ya referido y, en cambio, cobran relevancia 'la cuestión del pluralismo' y el asunto de 'la estabilidad' para la justicia como equidad 5 .

Volviendo al primer Rawls, es necesario comenzar enfatizando el modo como él eleva la justicia a "primera virtud de las instituciones sociales, como la verdad lo es de los sistemas de pensamiento" (Rawls, 1997). La sociedad es, en último análisis, un sistema de cooperación social y Rawls asume que ninguna sociedad es políticamente viable si sus miembros no saben cuáles son los principios que en ella rigen la distribución de cargas y beneficios o, sabiéndolo, no están de acuerdo con estos. De ocurrir un desconocimiento o un acuerdo con esas características, esa no sería una sociedad políticamente viable. Ahora bien: la justicia, como virtud, no es algo que deban acreditar los individuos, o alguna institución en particular, sino la sociedad como un todo, a través de eso que el filósofo llama la 'estructura básica de la sociedad', es decir, esos arreglos institucionales con los que nos encontramos al momento de nacer y que determinan buena parte de nuestra vida aun antes de que nosotros, de una manera libre, podamos definirle una meta. Como parte de esa estructura, al lado de los poderes públicos, del mercado, del mundo educativo y laboral, está el sistema de salud y de esa manera, por supuesto, debe ser sometido, como toda la estructura, al imperio normativo de los dos principios de justicia en que su teoría, llamada Justicia como Equidad, se sintetiza.

El primero prescribe que cada persona tiene un "derecho comparable a disfrutar del sistema más extendido de libertades básicas iguales para todos, compatible con un sistema para todos”. En otras palabras, que nadie pueda disfrutar de más libertad que los otros; por eso se lo conoce como el 'principio de la igual libertad'; al fin y al cabo, prescribe la igualdad (igualdad de la libertad). El segundo, en cambio, se ocupa de las desigualdades económicas y sociales, que solo serán admisibles si cumplen dos condiciones: a) deben ser para el mayor beneficio de los miembros menos aventajados de la sociedad $y \quad b$ ) deben asociarse a funciones y posiciones abiertas a todos, en condiciones de igualdad equitativa de oportunidades. Rawls pretende que con esos dos principios se concilian tres valores caros a la Modernidad política (como quiera que fueron la consigna de la Revolución francesa): Libertad, Igualdad y Fraternidad (solidaridad social). El segundo principio protege la igualdad en la forma de 'igualdad de oportunidades' y trata de alcanzar la solidaridad en tanto que recompensa a quienes contribuyan al mejoramiento de los 'menos aventajados' (Rawls, 1997). En tanto que se orientan de esa manera a la estructura básica de la sociedad, tienen una significación política en el sentido de que orientan las que arriba se llamaron 'soluciones políticas de una más radical naturaleza’.

5 Hay múltiples estudios sobre los giros que van del primero al segundo Rawls. Pero puede también verse en Grueso (2008). 
No es este el lugar para explicar el modo en que, una vez presentada la teoría en Una teoría de la justicia (1971), Rawls recibió múltiples críticas e inspiró teorías alternativas de justicia, unas de filiación liberal (Ronald Dworkin, Bruce Ackerman, Robert Nozick), otras comunitaristas (Michael Sandel, Charles Taylor, Michael Walzer), otras socialistas (Philippe Van Parijs, Milton Fisk), por solo citar algunos autores. Esto solo se menciona para ubicar el Enfoque de las capacidades, desarrollado por el filósofo y economista indio Amartya Sen y luego complementado por la filósofa estadounidense Martha Nussbaum, como una de esas teorías alternativas. Y también señalar que es en relación con la libertad que Amartya Sen toma distancia de Rawls. Por ende, no sobra señalar algunas peculiaridades en relación con la libertad en Rawls.

Como pensador liberal, Rawls pondera la libertad en alto grado y trata de garantizarla a través de su primer principio de justicia. Por supuesto, va más allá de defenderla como un derecho y defenderla, al modo de John Locke o de pensadores neoliberales contemporáneos, como el máximo de los derechos. Si ha de honrarse como algo que la justicia debe garantizar (y eso en buena parte es lo que su primer principio hace), debe ser conciliada con la igualdad de oportunidades. A los individuos, en virtud de esa primacía de la libertad, se les debe garantizar el derecho de gozar libremente de lo que su esfuerzo y su libertad de elección le han abierto como oportunidades en la vida. Solo que los individuos en las sociedades liberales están lejos de hacer parte de un sistema en donde haya igualdad de oportunidades, con lo que las suertes individuales no pueden generar el resultado del trabajo y del mérito individual. Las sociedades liberales deberían ser meritocráticas y, en cambio, están lejos de serlo, en virtud de las desigualdades sociales, de las formas como las clases sociales y las familias ya diferencian a los individuos al momento de nacer. Y también lo están por efecto de la 'lotería natural', pues también al momento de nacer, como lo hacen las familias y las clases (y la 'raza', el género y la orientación sexual, en virtud del racismo, el machismo o la homofobia existente en las sociedades), la naturaleza 'nos desiguala', afectando nuestra libertad y el alcance de nuestras 'elecciones', en gran parte ya determinadas.

Rawls pretende que sus principios de justicia contrabalancean necesidades, capacidades y méritos, neutralizando tanto el efecto de los determinantes sociales como de la determinación de la 'lotería natural' o del encuentro entre nuestras determinaciones biológicas y los prejuicios propios del sistema simbólico de las sociedades.

Rawls, interesado en proteger la libertad a toda costa, comenzó tratando de garantizar la igualdad de esta entre todos los ciudadanos, así como, por otra razón, puso la 'igualdad de oportunidades' como condición para permitir la desigualdad económica y política. En ese orden de ideas entran en acción los que él llama 'bienes primarios', medios omnivalentes que están a disposición de los individuos para que estos puedan alcanzar los fines que se proponen y que les dan sentido a sus vidas. Son decididos, como los principios, en la 'posición original', todavía tras el 'velo de ignorancia', es decir, ese artificio argumentativo que el primer Rawls empleó para explicar la validez moral de sus principios. A través de esos bienes, la teoría busca garantizar libertades, medios de subsistencia, oportunidades, autorrespeto, etc. Esto había que mencionarlo pues es con referencia a estos bienes primarios que primero toma fuerza la toma de distancia de Amartya Sen. 
En su obra Inequality Reexamined, tomando en cuenta lo que se espera de esos medios, Sen termina perfilando una diferencia substancial en relación con la libertad rawlsiana. Relaciona los bienes primarios con las libertades reales.

Yo pienso que el particular foco informacional sobre el cual se concentra Rawls elude algunas consideraciones que pueden ser muy importantes para el aseguramiento de la igualdad (y también de la eficiencia). La importancia del contraste toma en consideración la diversidad de los seres humanos. Dos personas que tienen el mismo paquete de bienes primarios pueden tener muy diferentes libertades para tratar de realizar sus respectivas concepciones de lo bueno (sin importar si estas concepciones coinciden o no) [...]. La importancia práctica de esta divergencia puede ser tan grande cuando se trata de lidiar con las desigualdades relativas al género, a la ubicación, a la clase y a toda suerte de variaciones humanas heredadas. (Sen, 1995)

Allí donde Rawls creía resolver todo garantizando la igualdad de oportunidades, Amartya Sen cree necesario alcanzar la 'igualdad de capacidades'; eso es a lo que la justicia social debe propender, pues la capacidad decide, más determinantemente, esa libertad que Rawls quiso proteger: la libertad efectiva de los individuos. Un sujeto exhibe su libertad en la medida en que tenga mayor capacidad u oportunidad real para lograr (ser o hacer) aquello que tiene razones para valorar (Nussbaum y Sen, 1996). Las libertades para Sen se definen más bien en términos de las capacidades y estas, lejos de ser omnivalentes, pueden llegar a alcanzar valoraciones variables en contextos igualmente variables. Aquí está la clave del nexo entre capacidad y libertad que luego trataremos de vincular en el análisis de las personas en situación de discapacidad (Sen, 2001).

Antes de seguir, conviene señalar, aunque sea de paso, otro punto de desencuentro entre el enfoque de las capacidades y la teoría rawlsiana de la justicia; esta vez a cargo de Martha Nussbaum. Según ella, la teoría rawlsiana excluye a las personas en situación de discapacidad. En concreto, dice, al considerar los bienes primarios y su opción de medir las posiciones sociales relativas (una vez fijada la prioridad de libertad), Rawls hace referencia a los ingresos y la riqueza, en lugar de usar un principio más heterogéneo como las capacidades. Y Nussbaum señala eso remitiéndose a la naturaleza contractualista detrás de la 'justicia como equidad' (Nussbaum, 2007; Rawls, 1997).

Como una pista, que aquí no se desarrollará, solo conviene señalar que Nussbaum no toma en cuenta lo que antes se quiso señalar con la noción rawlsiana de 'lotería natural'. Quizás lo que le da algo de validez a la crítica de la filósofa es el hecho de que Rawls no ahondó sistemáticamente en esa noción. De esa manera, por ejemplo, podría haber desarrollado una más informada y focalizada perspectiva en relación con la discapacidad como fenómeno de injusticia social. Se podría añadir, un poco también en defensa de Rawls, que a pesar de que dijo, desde el comienzo, que apelaba al modelo del Contrato Social (desarrollado a principios de la Modernidad por Hobbes, Locke y Rousseau) para fundamentar su teoría de la justicia, lo que más bien hace cuando deriva su teoría de la posición original es utilizar la Teoría de los Juegos y las teorías de decisión racional bajo condiciones de incertidumbre para avanzar un modo kantiano de afirmar la justicia (Grueso, 1997). Juntando las dos cosas que se acaban 
de decir, se podría someter a crítica la propia crítica de Nussbaum; aquella según la cual una teoría de la justicia de filiación contractualista es incapaz de tomar en cuenta la deficiencia física o mental. Que a las personas en discapacidad “... no se las trata como iguales a los demás ciudadanos. Sus voces no son escuchadas cuando se eligen los principios básicos...” (Nussbaum, 2007). En la posición original, tras el velo de ignorancia, los contratantes no saben si sufren alguna condición de discapacidad y terminan 'legislando' también a favor de esa condición, porque puede ser ese su caso en la vida real. De nuevo, una vez más, lo que es criticable en Rawls es que no se haya detenido lo suficiente en esta consideración.

En resumen, la condición de discapacidad entra, necesariamente, bajo la exigencia de alcanzar una igualdad de oportunidades y, en última instancia, tanto el sistema educativo como el sistema de salud deben garantizarlo. Pero no deja de ser menos cierto que Rawls no le dedicó suficiente atención al asunto de la discapacidad como una forma de injusticia social. Así que razón parcial le asiste a Nussbaum cuando, al remitirse a Kant, a su concepción de la persona moral, y poner en evidencia las condiciones óptimas que ella supone, trata de involucrar también a Rawls, al fin y al cabo, un kantiano. Esta noción de persona parece estar detrás de su listado de bienes primarios de Rawls y a Nussbaum le parece que ese listado supone una persona con un grado elevado de racionalidad, moral y prudencial, lo cual excluye a las personas con deficiencias mentales principalmente, que en virtud de esas condiciones más bien quedarían al margen de los que se supone que son los ciudadanos libres e iguales. En la perspectiva de Nussbaum, es mandatorio que el Estado asegure un umbral aceptable de capacidades, especialmente en las personas cuyas capacidades están por debajo del umbral funcional.

Dicho eso, procedamos a examinar el enfoque de las capacidades en relación con la discapacidad, ya más ortodoxamente en su versión seniana.

\section{Capacidad, capacidades, funcionamientos y libertad}

Sen cambia un poco la función que cumplen los bienes primarios en la teoría rawlsiana de la justicia y evita preguntarse por el grado de satisfacción de las personas o por la cantidad de recursos con que cuentan para llevar un tipo de vida u otra. Su orientación se enfoca, ante todo, hacia lo que las personas son capaces de hacer o ser realmente (Rawls, 1997; Sen, 2001). La idea básica es que el concepto de capacidades brinda una mejor perspectiva de las ventajas individuales, si se compara con lo que ofrece la teoría rawlsiana de los bienes primarios.

Bien podríamos quedarnos en el ámbito de los bienes primarios como lo propone Rawls -admite Sen- si no fuera porque existe una diversidad en la especie humana, con necesidades muy distintas, en contextos muy variados, con una pluralidad de intereses que hace que la conversión de bienes en capacidades cambie de una persona a otra.

El concepto de capacidades surge entonces ligado a las exigencias de la igualdad en la discusión de la filosofía política y de la preocupación de Sen por la igualdad de 
bienes primarios, manifestada previamente por Rawls. Esa es básicamente la corrección que Sen desarrolla en Inequality Reexamined. Allí buscaba evaluar y valorar el bienestar desde el punto de vista de la habilidad de una persona para hacer actos o alcanzar estados valiosos y de esta manera llega al término capacidades, que se centra en las distintas combinaciones alternativas que una persona puede hacer o ser, lo que más tarde llamará los funcionamientos que se pueden lograr (Nussbaum y Sen, 1993). $\mathrm{Su}$ aporte, en este sentido, no es otro que un desplazamiento de la atención de los bienes primarios a lo que los bienes suponen para las personas, es decir, lo que ellas pueden hacer con esos bienes. Emerge allí un concepto novedoso: la "igualdad de capacidad básica"6.

Al concepto de 'capacidades' se une el de 'funcionamiento', que no está relacionado solo con el desarrollo de una tarea o una actividad, sino con un 'estado'. Ejemplos de funcionamientos es ser feliz o estar libre de enfermedad. Así el concepto se extiende hasta incluir, desde la visión de Sen, rasgos personales o que permiten su expresión. La vida, entonces, es un conjunto de funcionamientos que se encuentran interrelacionados, consistentes en estados y acciones. Ese conjunto es de tal importancia que la realización de una persona puede entenderse como la suma de sus funcionamientos, ya que estos son las peculiaridades del estado de su existencia, reflejando así las diversas cosas que puede hacer o ser y el bienestar con el que una persona cuenta para vivir en sociedad.

Con eso en mente, podemos volver a la noción de 'capacidades', entendida como un conjunto de funcionamientos o una suma de funcionamientos, que reflejan la libertad de la persona para alcanzar aquello que valora. Una capacidad, en otras palabras, no es más que las diversas combinaciones de funcionamientos que se pueden conseguir, como la habilidad para estar bien nutrido, tener buena salud y la posibilidad de escapar de la mortalidad evitable y prematura.

La capacidad proporciona un punto de vista desde el cual valorar las condiciones sociales, políticas y económicas que viven las personas al interior de la sociedad. Estas condiciones son importantes porque pueden llevar al desarrollo o al deterioro de los funcionamientos y las capacidades de la persona. La ausencia o el deterioro de la capacidad individual es una muestra de desigualdad, ausencia de bienestar y una precaria calidad de vida que se puede vivir en la sociedad.

Llegados a este punto, conviene volver, aunque solo sea de manera momentánea, a Martha Nussbaum. Ella comparte la idea general de que las capacidades se refieren a "lo que es capaz de hacer y de ser una persona" (Nussbaum, 2012). Comparte también la creencia seniana de que el "espacio de capacidad" es el lugar adecuado para evaluar la calidad de vida de las personas y las desigualdades entre las personas. En relación con el funcionamiento se mantiene en aquello de que se refiere a la "realización activa

6 El giro que hace Sen alcanza también para dejar notar por qué en Rawls la ocupación con los bienes primarios 'extravía' la atención que, en otras condiciones, merecería la salud como un problema de justicia. Dice: "En principio, la equidad en salud puede tener alguna importancia, pero que es absorbida empíricamente por la atención que debemos prestar a la distribución de los recursos o 'bienes primarios', dado que son estos recursos económicos y sociales los que, en última instancia, determinan el estado de salud de las personas" (Sen, 2002). 
de una o más capacidades" (Nussbaum, 2012) y, al igual que Sen, le concede mayor importancia a las capacidades sobre los funcionamientos, debido a que "garantizan la existencia de un espacio para el ejercicio de la libertad humana" (Nussbaum, 2012). Hasta allí hay coincidencias. Pero va un poco más allá y profundiza lo avanzado por Sen, incluso tratando de alcanzar unos principios generales de justicia. Identifica diez "capacidades humanas centrales" y agrega los componentes de dignidad y liberalismo político, muy en una línea kantiana y rawlsiana (a pesar de criticar a Rawls) de un modo no presente en la teorización de Sen (Nussbaum, 2007).

Tras esta sumaria revisión del enfoque, conviene ahora volver al modo en que las capacidades involucran la libertad de las personas de elegir entre diferentes estilos de vida, para que así el enfoque pueda sernos útil en el entendimiento de la discapacidad como un asunto de justicia social. En este punto es importante traer a colación, diferenciándolo de Rawls, el modo en que Sen involucra más decididamente la salud con la libertad y la justicia, permitiendo ir más allá de Rawls en la concreción de la equidad. Dice:

La equidad en salud puede formar parte integral del marco más amplio de la equidad en general, pero siempre habrá algunas consideraciones especiales relacionadas con la salud que forzosamente tendrán que integrarse en la evaluación de la justicia en general. Al hacer este ejercicio, la idea de equidad en salud plantea algunas cuestiones y perspectivas específicas que enriquecen la noción más abstracta de la equidad en general. (Sen, 2002)

Sen ve la capacidad como el espacio en el cual se desarrollan diferentes condiciones de vida de las personas si se quiere una sociedad verdaderamente libre e igualitaria (Sen, 2001). La capacidad entonces es concebida por Sen como un sentido de Libertad:

La "capacidad" de una persona se refiere a las diversas combinaciones de funcionamientos que puede conseguir. Por lo tanto, la capacidad es un tipo de libertad: la libertad fundamental para conseguir distintas combinaciones de funcionamientos (o, en términos menos formales, la libertad para lograr diferentes estilos de vida). (Sen, 2001, traducción libre)

Así, la libertad, desde el enfoque de capacidades planteado por Sen, es un aspecto indispensable para el logro del bienestar y de la calidad de vida tanto de los individuos

7 Las diez capacidades centrales establecidas por Nussbaum, en su libro Las fronteras de la justicia son: i) vida; ii) salud física; iii) integridad física; iv) sentidos, imaginación y pensamiento; v) emociones; vi) razón práctica; vii) afiliación; viii) otras especies; ix) juego, y x) control sobre el propio entorno.

8 Todavía en relación con Nussbaum, habría que agregar que para ella las capacidades básicas, las capacidades internas y las capacidades combinadas se constituyen en las tres categorías de capacidades que, a diferencia de Senn, Nussbaum desarrolla de manera jerárquica. En las capacidades combinadas Nussbaum resalta el papel de las condiciones externas; para su desarrollo, según Nussbaum "Las capacidades combinadas" son capacidades internas que asociadas con las adecuadas condiciones externas le permiten a la persona ejercer una función; así, por ejemplo, en el caso de una persona analfabeta que vive en condiciones de pobreza excluida de una educación elemental, presenta capacidades básicas e internas, pero carece de capacidades combinadas para acceder a un empleo digno y entrar en el ámbito de la producción (Nussbaum, 2012). 
como de las poblaciones. Nunca se enfatizará suficientemente esto: sin libertad no hay logro de bienestar, ni conformidad con el modo de vida que terminamos viviendo, individual y colectivamente. Todo esto, llevado al campo de la salud como campo del que debe ocuparse la justicia, queda plenamente establecido por Sen en su artículo de 2002 “¿Por qué la equidad en salud?”:

Un enfoque adecuado de la política de salud debe tener en cuenta no solo las influencias de los factores sociales y económicos generales, sino también una gran variedad de parámetros distintos, como las discapacidades personales, la propensión individual a la enfermedad, los riesgos epidemiológicos de cada región en particular, la influencia de las variaciones climáticas, etc. Una buena teoría de la equidad en salud tiene que darles a estos factores su valor dentro de la disciplina de la equidad en salud. En general, al hacer una política de salud es necesario distinguir entre la igualdad en los logros de salud (o las correspondientes posibilidades y libertades) y la igualdad en la distribución de lo que, en términos generales, se pueden llamar recursos sanitarios. (Sen, 2002)

Quizás ayude a fijar esta idea el ejemplo dado por Mario Toboso y María Soledad Arnau, quienes comparan la situación de una persona que pasa hambre con la de otra que ayuna. Si solo nos atuviéramos a los funcionamientos, se podría decir que no hay diferencias, pues existe en ambos casos un logro no alcanzado: obtención de alimento. Pero al analizarlo desde el punto de vista de la libertad se visualiza la diferencia fundamental. La primera persona no tiene otra opción que pasar hambre, en tanto que la segunda ha elegido libremente ayunar. La persona que ayuna ha elegido libremente esa opción entre otras diferentes opciones, mientras que la otra persona no ha tenido posibilidad de elegir (Toboso y Arnau, 2008). Este aspecto es importante porque tiene en cuenta, además de los funcionamientos reales de un individuo, las elecciones tomadas como elemento fundamental para evaluar el bienestar.

La cuestión arroja nuevos matices si se toma en cuenta la capacidad como libertad en relación con la discapacidad. Por un lado, se encuentran los sujetos con discapacidad mental, con alto compromiso de su libertad, quienes dependen de otros sujetos para la toma de decisiones en relación con su estado de bienestar; por otro lado, están los estigmas sociales que pueden existir en relación con la capacidad de elección que tienen aquellas personas con discapacidad, pero con su capacidad de elección intacta; es decir, la creencia de que tener $x$ o $y$ condición de discapacidad ya genera una pérdida de la libertad; y finalmente se encuentran las personas con discapacidad motora y sensorial importante, que necesitan constantemente la ayuda de otras personas para realizar a diario sus actividades para alcanzar los logros relacionadas con el bienestar.

En estos casos, el requerimiento constante de un cuidador, sumado a la sobreprotección familiar y a los diferentes prejuicios sociales, generan de manera constante una confusión entre la ayuda requerida para el alcance de un logro (funcionamiento) y la invasión en la capacidad (libertad) del sujeto. Aquí el dilema es hasta qué punto el nivel de ayuda o apoyo social ofrecido por el sistema social favorece la libertad o por el contrario limita la capacidad total de un individuo para conseguir que los funcionamientos alcanzados sean mejores; asunto difícil de abordar. 
Así, los planteamientos del Enfoque de Capacidades de Sen permiten entender cómo, al ser las capacidades un asunto de libertad, la discapacidad emerge como un déficit de libertad de elección de un sujeto con una alteración corporal en relación con diferentes condiciones de su vida que alimentan o comprometen su bienestar. Esta limitación puede ser generada por condiciones económicas, políticas y sociales y por las relaciones entre estas, que han sido impuestas a lo largo de la historia, pero que, hay que reconocerlo, han venido transformándose, y por condiciones individuales propias del contexto específico de cada sujeto.

\section{La discapacidad desde el enfoque de las capacidades}

Dos experiencias de rehabilitación pueden servir para ilustrar lo que se quiere decir con este abordaje de la discapacidad, como un asunto de justicia social, desde el enfoque de las capacidades: la de Pedro y la de Juan.

Pedro, joven de 25 años, asiste a un centro de rehabilitación, seis meses después de haber sufrido una herida por arma de fuego a nivel de la columna, ocasionándole un trauma raquimedular a nivel de T4 y dejándolo parapléjico. Se trata de un ingeniero de sistemas egresado de una universidad privada y que desempeña su rol profesional en una empresa familiar de plásticos, en la cual el gerente general es su padre. Es adherente a su proceso de rehabilitación y asiste a este generalmente en compañía de su novia de 21 años, estudiante de Medicina, cuando no va acompañado de su madre o hermana. Pedro logró reintegrarse laboralmente a los dos meses de su egreso hospitalario. Adicionalmente, en las tardes, tres veces a la semana, asiste al club, en donde recibe clases de waterpolo; actividad que realiza desde los 12 años y que reinició contratando un profesional experto que tomará en cuenta su condición.

A la misma institución de salud a que asistió Pedro, asistió Juan, hombre de 37 años, quien, en el contexto de un accidente de tránsito, sufrió un trauma raquimedular a nivel de T4, ocasionándole también una paraplejía. Juan se desempeñaba, antes de su accidente, como distribuidor y vendedor de tenis y ropa en el centro de la ciudad. Es padre de una bebé de 2 años, con quien no convive, pero de quien se hace responsable. Él vive con sus padres, ambos adultos mayores, y es poco adherente al proceso de rehabilitación, tanto que hay semanas en las que no asiste a una sola sesión de terapia. No ha podido reiniciar su vida laboral.

¿Por qué respuestas tan diferentes a la terapia? ¿No presentan Pedro y Juan las mismas incapacidades considerando que, a nivel orgánico y/o sistémico, son diagnosticados de la misma forma por el sistema de salud y orientados a seguir el mismo proceso de rehabilitación?

De alguna forma, Juan y Pedro no poseen las mismas capacidades, a pesar de la clasificación que, tras su evaluación, hizo de ellos el sistema de salud. En cada caso toman relevancia las situaciones diferenciadas del día a día, el peso del acceso y uso diferenciado de los servicios de salud -si bien esto solo da cuenta de un componente pequeño de la concepción de bienestar-y de otras condiciones estructurales de índole social y económico. Y es con base en todo esto que puede llegar a decirse que hay en- 
tre Juan y Pedro diferencias importantes en la capacidad de hacer y ser como persona. Ambos, es cierto, presentan la misma condición anatomo-fisiológica limitada a nivel de los órganos del movimiento y sensoriales, así, a la luz de la jerarquía de capacidades desarrollada por Nussbaum, Pedro y Juan parecen presentar similitud en sus capacidades básicas, las cuales son la base necesaria para el desarrollo de capacidades más avanzadas, pero difieren sustancialmente en sus capacidades combinadas, las cuales, según Nussbaum, son aquellas capacidades internas que en asocio con adecuadas condiciones externas le permiten a la persona ejercer una función; así, por ejemplo, la capacidad de movilidad, capacidad que, bajo el enfoque de Nussbaum, hace parte de la integridad física, es mayor para Pedro al depender esta de las condiciones sociales y económicas propias del contexto de cada individuo (Nussbaum, 2012).

Hasta donde se puedan mantener como universales algunas capacidades, habría que defenderlas o propiciarlas con independencia (relativa) del contexto de aplicación. Así las cosas, Juan y Pedro deberían ser evaluados de igual manera en términos de capacidades, a pesar de que, en un mismo espacio geográfico (la misma ciudad), sus contextos económicos y sociales sean diferentes. Al ser tan entrelazados los conceptos de capacidad y funcionamiento en el enfoque que se ha expuesto, se dificulta bastante diagnosticar si lo que obra en esta respuesta diferenciada al tratamiento y formas de rehabilitación son las capacidades o el funcionamiento de los individuos en cuestión.

Si las capacidades pueden ser consideradas universales, es decir, si son independientes del contexto, y siguiendo el enfoque de Nussbaum, es pertinente que Juan y Pedro sean evaluados en términos de estas capacidades básicas similarmente; este pensamiento se constituye en un núcleo fundamental para entender, en este ámbito, los conceptos de justicia e igualdad; aspectos que se retomarán al final de este documento.

Al lograr Pedro desplazarse en silla de ruedas, acceder a diferentes espacios públicos y privados con infraestructuras adecuadas, fortalecer sus extremidades superiores, etc., está dando cuenta de funcionamientos específicos (logros alcanzados) que alimentan su capacidad de movilidad y que probablemente representen en él un nivel de discapacidad menor en relación con la de Juan, quien presenta funcionamientos comprometidos que limitan el desarrollo de su capacidad de movilidad y, por lo tanto, mayor discapacidad.

Pedro desarrolló su capacidad de movilidad porque tuvo la libertad de escoger movilizarse en espacios con infraestructuras adecuadas, tuvo la libertad de escoger una adecuada silla de ruedas y libertad de ser adherente a su proceso de rehabilitación (o, por lo menos, estaba en una situación en la que tomar esa oportunidad se hacía mandatoria de una manera que, al parecer, no estaba abierta para Juan). Podemos suponer que, a diferencia de Pedro, a Juan le correspondía desplazarse en espacios con infraestructuras no adecuadas; se movilizaba en la silla de ruedas dada por su entidad de salud, y bajo las condiciones que ese tipo de entidades imponen, y no asistía al proceso de rehabilitación por aspectos económicos (no tener para el taxi) o sociales (no tener quien lo acompañe), condiciones que le impiden el desarrollo de su capacidad de movilidad. $\mathrm{O}$, simplemente, no estaba en una situación que, teniendo en cierto sentido la opción de rehabilitarse, e incluso siendo también para él mandatoria en algún sentido, todo jugó a favor de eludir esa oportunidad y traicionarse a sí mismo pospo- 
niendo el proceso de rehabilitación. Quizás se pueda decir que, más allá de la igualdad y libertad individual dentro del sistema de seguridad social del que goza Juan, él era en verdad menos libre que Pedro: menos libre para rehabilitarse, así tuviese a la mano la misma oferta médica y de rehabilitación; menos libre para adherirse a un proceso que lo sacara adelante. Que otras cosas terminaron pesando para hacerle más dependiente de otras personas o más limitado su campo de acción. Que todo eso concurrió para ahondar su condición de discapacidad.

Esta 'menor libertad' está dada en función de las oportunidades educativas, oportunidades laborales, económicas y sociales; distribuidas diferencialmente entre Pedro y Juan y que se constituyen en los determinantes sociales de cada uno, es decir, en las condiciones en las que Pedro y Juan nacieron, crecieron, viven y se envejecen; condiciones que son diferentes para los dos sujetos y que terminan socavando condiciones marcadas de desigualdad en salud y que, por lo tanto, restringen la participación en la sociedad para el caso particular de Juan.

Tanto Pedro como Juan podrían tener acceso a un mismo proceso asistencial de salud y rehabilitación, pero es evidente que hay una desigualdad en el aprovechamiento de los mismos y es en este aspecto en el que se llama la atención. A este punto, el análisis requiere desplazarse de los casos particulares a las políticas públicas, para ver si estas están en capacidad de remover esos determinantes sociales que determinan la distribución desigual de funcionamientos que vemos entre Pedro y Juan.

Aquí cobra relevancia la cuestión, ya avanzada por Sen, de que la equidad en salud no puede analizarse solo a través de la igualdad en el acceso a los servicios sanitarios. Si esa igualdad se garantiza de forma aislada, dejando intocadas las profundas asimetrías sociales, no habríamos ganado mucho en materia de justicia social, incluso si estos dos individuos, cada uno por sí mismo, obtuvieran una mejoría acorde a sus condiciones socioeconómicas al margen de su igual acceso a la atención médica. En relación con Pedro y Juan es posible extrapolar lo que dice Sen con relación a dos individuos, A y B, y la equidad en salud'.

\footnotetext{
9 "Supongamos que las personas A y B tienen exactamente las mismas predisposiciones desde el punto de vista de la salud, entre ellas la misma propensión a una enfermedad particularmente dolorosa. Pero A es muy rico y consigue curar o suprimir completamente su dolencia con algún tratamiento médico caro, mientras que $\mathrm{B}$ es pobre y no puede pagarse ese tratamiento, por lo que sufre mucho con la enfermedad. Aquí hay una clara desigualdad en la salud. Además, si desde el punto de vista moral no aceptamos que el rico reciba un tratamiento privilegiado, se puede argumentar que también hay una violación de la equidad en salud. Concretamente, los recursos usados para curar al rico A podrían haberse usado para proporcionarle algún alivio a ambos o, en caso de indivisibilidad, para proporcionarle a ambos la misma probabilidad de obtener la curación a través de algún mecanismo probabilístico. No es difícil defender esta argumentación.

Ahora imagínense un cambio de política introducido por algún defensor de la igualdad sanitaria que le dé prioridad a la reducción de las desigualdades sanitarias y que le impida al rico A comprar una curación que el pobre B no pueda comprar. La vida del pobre B no se habrá visto afectada, pero ahora el rico A también vivirá con la dolorosa enfermedad y gastará su dinero, digamos que en viajes consoladores en un lujoso yate en mares exóticos. El cambio de política ha reducido, de hecho, la desigualdad en salud, pero ¿se puede decir que haya mejorado algo la equidad en salud?” (Sen, 2002).
} 
6. Ventajas y limitaciones de aplicabilidad poblacional del enfoque de las capacidades en salud pública

Lo que está ahora en cuestión es el alcance del enfoque de las capacidades para orientar políticas públicas en materia de salud; políticas capaces de neutralizar los efectos de condiciones extraterapéuticas sobre el aprovechamiento de los dispositivos y los recursos de la salud, pues hemos supuesto que esas condiciones están por fuera de la atención en salud en sí misma.

De alguna forma, el enfoque de capacidades nos invita a centrar la mirada en lo que los individuos pueden realmente llegar a ser y hacer en sus vidas, sin descuidar los factores que pueden contribuir a los logros y fracasos en el campo de la salud y que van mucho más allá de la atención sanitaria: "Las predisposiciones genéticas, los ingresos individuales, los hábitos alimentarios y los estilos de vida hasta el entorno epidemiológico y las condiciones de trabajo". Ha dicho que "la equidad en salud no puede preocuparse únicamente de la desigualdad en la salud o en la atención sanitaria, y debe tomar en consideración cómo se relaciona la salud con otras características a través de la asignación de recursos y de los acuerdos sociales” (Sen, 2002, p. 304). Pero, en general, la mirada se centra en el uso o conversión de dichos bienes en modos de ser o en realizaciones en el hacer.

Thomas Pogge descree un poco de esa perspectiva al manifestar que no existe en realidad mucha diferencia entre centrarse en los recursos o bienes y centrarse en las capacidades. Puesto que los bienes son valorados por el hecho de crear oportunidades para que las personas realicen sus planes de vida y la distribución de los mismos puede darse según las necesidades de los individuos (Pogge, 2002). Así, por ejemplo, para Pogge, una Teoría de recursos sofisticada puede tener un alcance semejante al enfoque de capacidades; debate que se complejiza al considerar que los bienes o recursos no son solo físicos, sino que incluyen bienes sociales, éticos, tales como libertades, oportunidades o bases sociales, y al considerar que la Teoría de Recursos ofrece varias ventajas, según lo planteado por Pogge, como el permitir la transparencia publica, la capacidad de medición y una clasificación o priorización; virtudes de las que carece el enfoque de capacidades (Pogge, 2002).

El enfoque de capacidades también difiere en el uso de los indicadores poblacionales agregados, que promedian el supuesto estado de salud de los individuos dentro de sus grupos poblacionales, sin tener en cuenta las desigualdades interindividuales, en las que los individuos realmente pueden ser y/o hacer; indicadores que han sido usados para la toma de decisiones en salud pública a nivel mundial y en los diferentes países.

Aquí, surge la duda de si la estrategia para la aplicación del enfoque de capacidades en términos poblacionales consiste en apuntar a maximizar las capacidades de grupos grandes, grupos poblacionales, sacrificando la mejora de las capacidades de unos pocos. Nussbaum pareciera tener una respuesta más clara, y está relacionada con el considerar la garantía de la suficiencia de las capacidades a un nivel umbral como una prioridad sobre la maximización de las capacidades totales o promedio de una población (Nussbaum, 2012). 
Pero esto conduce a un dilema complejo que es el de determinar qué hacer en una situación en la que el esfuerzo y los recursos necesarios para llevar a un individuo por encima del umbral de suficiencia podrían usarse alternativamente para expandir las capacidades de muchos individuos en un escenario en donde todos están por debajo del umbral de suficiencia y la elección es entre ayudar a que una persona llegue al umbral de suficiencia, frente a los de dos personas o más cerca del umbral. El dilema es entre obtener el mayor beneficio ayudando a uno o ser más justos a dos o más, pero con menos beneficio.

Así, al parecer, la aplicabilidad del enfoque de las capacidades para la toma de decisiones en salud pública, para abordar fenómenos complejos de salud, como la discapacidad y la salud mental, no es claro a nivel poblacional; y los autores de este enfoque no dieron una guía para su aplicabilidad.

Un segundo escenario que le plantea Singer a Nussbaum es el siguiente: "En el segundo escenario, hay una opción entre gastar enormes recursos para mejorar las capacidades de solo un individuo solo un poco para alcanzar el nivel de suficiencia, mientras que todos los demás están por encima del umbral y pocos recursos aquí podrían mejorar sustancialmente sus capacidades”. Según Singer, la aplicabilidad del enfoque de capacidades no es clara, y para tomar decisiones en términos poblacionales se presenta un "problema de agregación" o problema de "monstruo de recursos", en donde no es claro cómo direccionar la distribución de los recursos. Para Singer, si bien el enfoque de capacidades puede tener argumentos sustanciales para centrarse en las capacidades, en realidad no ofrece soluciones claras (Singer, 2002).

Así entonces, son innumerables los escenarios que se pueden presentar, en donde el abordaje de fenómenos complejos en salud, como la discapacidad, no es fácil de determinar. Estos escenarios requieren de una evaluación a través de las dimensiones, causas, niveles, consecuencias y posibles respuestas; y para estos el abordaje desde un enfoque interdisciplinario y transdisciplinario es fundamental. Sin embargo, en el proceso de construcción del conocimiento del abordaje de estos fenómenos y en la definición del tipo de intervención que se requiere se pueden cometer errores morales; es decir, considerar invertir recursos sociales en una población, por $X$ o $Y$ condición, excluye la posibilidad de que la justicia social exija que los recursos se gasten tal vez en un mínimo de la población en relación con las causas y las consecuencias de la discapacidad, por ejemplo. El enfoque de capacidades, como está planteado, motiva a considerar a nivel poblacional las condiciones en salud como problemas o condiciones multidimensionales, más que solo pensar en los intereses de unos pocos frente a los muchos o viceversa; sin embargo, no es clara.

\section{A manera de conclusión}

Sobra insistir en el nexo ideal entre las políticas públicas y la categoría normativa de la justicia social. En relación con la población en condición de discapacidad ese nexo sugiere un tipo específico de políticas orientadas a ampliar su horizonte de libertad; en concreto, ampliar los límites del margen de maniobra que los individuos tienen 
para elegir entre varias opciones; la libertad de darle sentido a la vida o de vivir su vida de acuerdo con su anhelo de libertad. Bien sabemos que lo que es válido para analizar el campo de libertad que alcanza un ser humano se torna limitado una vez caemos en las comparaciones interpersonales. Pero los seres humanos deberían ampliar el Juego de la Libertad de forma tal que varios modos de ser libre y, consiguientemente, plenamente humanos quepan en este. Es a esto a lo que apuesta en gran parte el enfoque de las capacidades, del que aquí hemos recuperado algunos elementos útiles para analizar el caso de la condición de discapacidad.

Pero, como bien se dijo desde la introducción, había que evaluar el enfoque en términos de su potencialidad para orientar políticas públicas que amplíen el juego de la libertad para un mayor número de personas. Ahí el enfoque parece perder potencialidad, como bien se ha tratado de mostrar hacia el final del artículo, especialmente por el más bien poco análisis del rol jugado por los otros determinantes sociales. Es allí donde entran a jugar un papel más decisivo otras perspectivas justicieras y un abordaje transdisciplinario que dé cuenta de cómo juegan las diferentes determinaciones sociales en las vidas individuales (Méndez, 2015). No de otra forma se llega a diseñar políticas públicas progresistas y justicieras. Lo impone el hecho de estudiar de manera detallada aspectos específicos de cada sociedad; especialmente aquellos que determinan, a partir de una discapacidad física o mental, una mejor libertad de algunos individuos.

\section{Referencias bibliográficas}

Daniels, N., Kennedy, B. y Kawachi, A. (2004). Health and inequality, or, why justice is good for our health, public health, ethics, and equity. Oxford University Press.

Elster, J. (1997). El estudio empírico de la justicia. En D. Miller y M. Walzer (Comps.), Pluralismo, justicia e igualdad. Fondo de Cultura Económica.

FIsk, M. (2000). Toward a bealthy society. The morality and politics of American bealth care reform. The Kansas University Press.

Grueso, D. I. (1997). Rawls: una hermenéutica pragmática. Universidad del Valle.

Grueso, D. I. (2008). La filosofía y la política en el pluralismo. Siglo del Hombre Editores.

MÉndeZ, F. (2015). Transdisciplina y la investigación en salud: ciencia, sociedad y toma de decisiones. Colombia Médica, 46(3), 128-135.

Nussbaum, M. C. (2007). Las fronteras de la justicia. Paidós.

Nussbaum, M. C. (2012). Crear capacidades. Paidós.

Nussbaum, M. C. y Sen, A. (1996). La calidad de vida. Fondo de Cultura Económica.

Pogge, T. W. (2002). Can the capability approach be justified? Philosophical Topics, 30(2), 167-228.

Rawls, J. (1997). Una teoría de la justica. Fondo de Cultura Económica.

SEN, A. (1995). La desigualdad reexaminada. Alianza Editorial.

SEN, A. (2001). Development as freedom. Oxford University Press.

SEN, A. (2002). ¿Por qué la equidad en salud? Revista Panamericana de la Salud, 11(5/6).

Singer, P. (2002). A response to Martha Nussbaum: reply to Martha Nussbaum, Justice for Non-Human Animals. The Tanner Lectures on Human Values. 
DISCAPACIDAD:

UN ASUNTO DE JUSTICIA SOCIAL

D. I. GRUESO VANEGAS Y L. M. SANDOVAL MORENO

ShKLAR, J. (2010). Los rostros de la injusticia. Herder Editorial, S. L.

Toboso, M. M. y Arnau, M. S. (2008). La discapacidad dentro del enfoque de capacidades y funcionamientos de Amartya Sen. Revista Iberoamericana de Filosofía, Política y Humanidades, 10(20), 64-94.

Venkatapuram, S. (2011). Health justice: an argument from the capabilities approach. Polity Press.

Walzer M. (1997). “La igualdad compleja” y "Seguridad y bienestar” en Esferas de la justicia. FCE. 


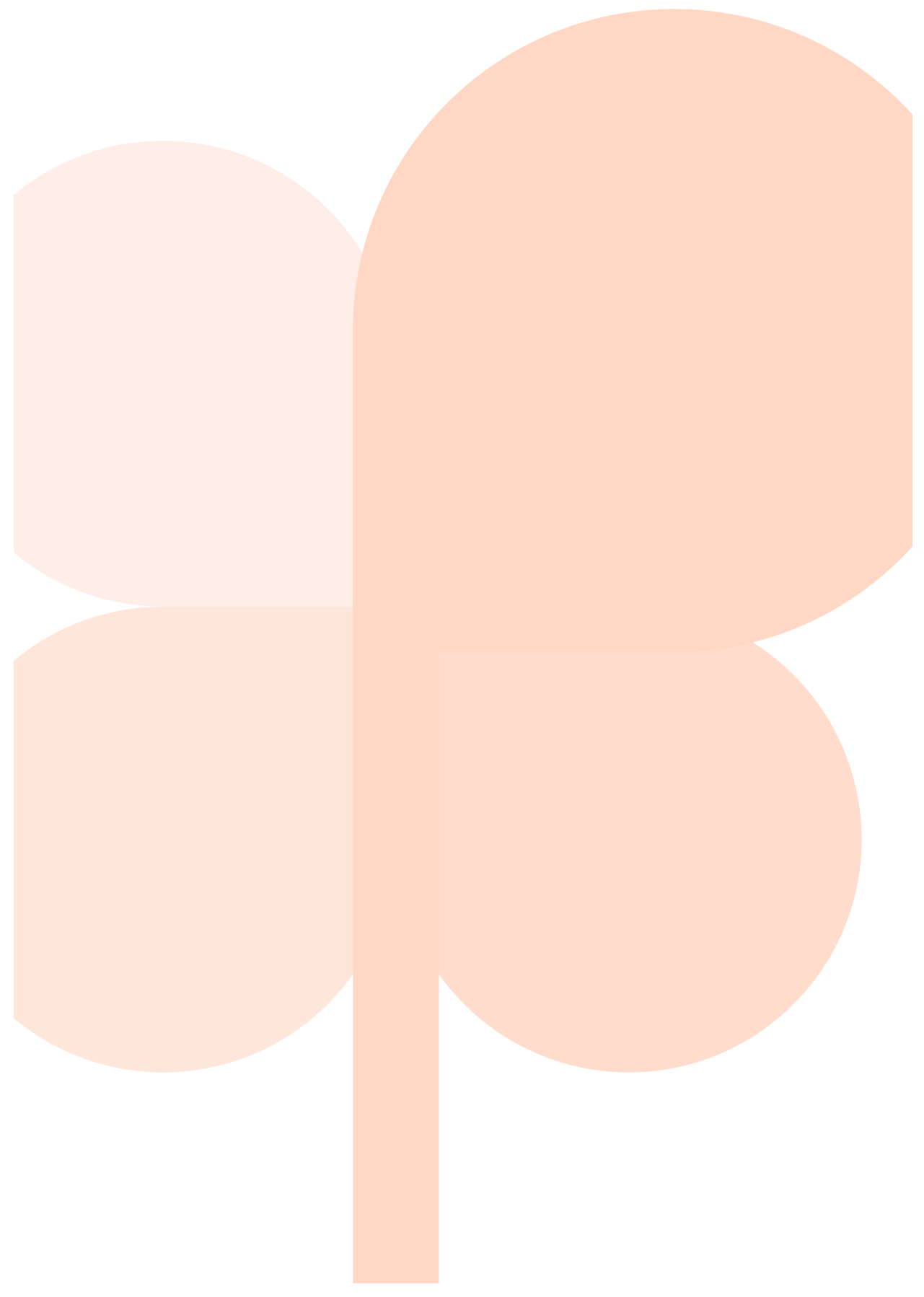

